\title{
Xanthogranulomatous inflammation in female pelvis: a diagnostic and therapeutic challenge
}

\author{
Pandey $\mathrm{D}^{1}$, Saxena $\mathrm{A}^{1}$, Rao ACK ${ }^{1}$, Valiathan $\mathrm{M}^{1}$, Thomas $\mathrm{J}^{1}$, Shetty $\mathrm{J}^{1}$
}

\section{ABSTRACT:}

Xanthogranulomatous inflammation is an unusual form of chronic inflammation that can present clinically with destructive or obstructive symptoms. It may also mimic malignancy at times. It is a rare entity as far as female genital tract is concerned. Histologically well characterized by a massive infiltration of the tissues by lipid-laden histiocytes, it usually poses diagnostic and therapeutic challenges for the clinician. We present here two interesting cases of the same, encountered by us.

Case 1: A 42-year-old lady with history of intra-uterine device (IUD) in situ for 8 years presented with a picture of acute on chronic pelvic inflammatory disease (PID). She was started on inpatient CDC regimen of PID. Despite this her symptoms persisted and based on high index of suspicion she was started empirically on anti-tubercular treatment (ATT). Three months later she presented again with similar complaints. Laparotomy picture was typical of abdominal tuberculosis and thus multiple biopsies were obtained. Histo-pathological report showed xanthogranulomatous inflammation. As there is no specific treatment for this condition, patient was continued with ATT. On three, six and twelve months follow up patient had been asymptomatic.

Case 2: A 48 year old lady with history of vaginal hysterectomy 6 months ago presented with right sided hydro-uretero nephrosis. CT scan showed this was because of external compression caused by an adnexal mass measuring $5.8 \times 3.9 \mathrm{cms}$. She underwent laparotomy with right oophorectomy. Right distal ureter was fibrotic so was excised and Boari flap was done. Histopathological diagnosis rendered was Xanthogranulomatous inflammation with abscess formation and chronic ureteritis with fibrosis.

Xanthogranulomatous inflammation of the genital tract is enigmatic disorder of unknown etiology. Gynecologist should be aware of this entity whenever the clinical presentation is atypical and not fitting into a diagnosis, or when the patient is not responding to conventional treatment of PID. Early diagnosis and management is very important as it can cause tissue destruction or may lead to obstructive disasters.

${ }^{1}$ Associate Professor, Department of OBG, KMC, MBBS, KMC, Manipal University, Manipal, Karnataka, India.

${ }^{1}$ Professor, Department of Pathology, KMC Manipal University, Manipal, Karnataka, India.

${ }^{1}$ Professor, Department of Pathology, KMC Manipal University, Manipal, Karnataka, India.

${ }^{1}$ Professor, Department of Urology, KMC Manipal University, Manipal, Karnataka, India.

${ }^{1}$ Additional Professor, Department of OBG, KMC, Manipal University, Manipal, Karnataka, India.

Correspondence: Dr Deeksha Pandey

Email: deekshiiiobg@gmail.com

Competing interests -none

\section{INTRODUCTION}

Xanthogranulomatous inflammation is an unusual form of chronic inflammation that can present clinically with destructive or obstructive symptoms. It may also mimic malignancy at times. More commonly seen in the urinary and gastrointestinal system, xathogranulomatous inflammation is a rare entity as far as female genital tract is concerned. Histologically well characterized by a massive infiltration of the tissues by lipidladen histiocytes, it usually poses diagnostic and therapeutic challenges for the clinician. We present here two interesting cases of the same, encountered by us in last one year.

\section{CASE 1}

A 42-year-old lady presented to us with fever, pain abdomen, diarrhea, and vomiting of 15 days duration. She had history of Intra-uterine device (IUD) in situ for 8 years, which was removed recently, when she went to a local doctor 5 days ago with the same complaints. She was started on broad spectrum antibiotics there. With that when she had no relief she presented to us. She was stable with BMI was... Examination revealed tenderness over left lumbar region and left iliac fossa. On pelvic examination left forniceal fullness was noted. Ultrasound showed heterogenous mass in the left adnexa measuring $6.4 \mathrm{~cm} \times 3.6$ $\mathrm{cm}$ with minimal ascitis and omental thickening. ESR was $126 \mathrm{~mm} / 1 \mathrm{~h}$ and tumor markers were mildly elevated (CEA: 12.67 ng/ml, CA-125: 69.07 U/ $\mathrm{ml}$ ). A preliminary diagnosis of acute on chronic pelvic inflammatory disease (PID) was made. She was started on inpatient CDC regimen of PID with injection clindamycin and gentamycin. Despite this her symptoms persisted and repeat ultrasound showed no decrease in size of mass. Ascitic fluid tap and endometrial sampling was done in order to find out if it was of tuberculous (TB) origin. Both samples however were reported negative for TB (acid fast bacilli, culture, polymerase chain reaction and culture). Still based on high index of suspicion she was started empirically on antitubercular treatment (ATT). On ATT, three months later she presented again with pain abdomen, diarrhea and vomiting. As on ultrasound same mass still persisted, a decision for its surgical removal and hysterectomy was taken. On laparotomy uterus and ovaries could not be visualized as the omentum was seen covering the abdominal and pelvic organs. Dense adhesions between greater omentum and intestines were seen. Peritoneum 
was thickened and tubercles of various sizes were seen scattered over the intestine, greater omentum and mesentery. Laparotomy picture was typical of abdominal tuberculosis so further procedure was deferred. Abdomen was closed after obtaining multiple biopsies. Once again there was no evidence of $\mathrm{TB}$ in these samples too, rather it showed xanthogranulomatous inflammation [Figure 2]. As there is no specific treatment for this condition, patient was continued with ATT. On three, six and twelve months follow up patient had been asymptomatic.

\section{CASE 2}

A 41-year-old lady presented with right sided abdominal pain for 6 months. She had undergone vaginal hysterectomy with bilateral salpingooophorectomy 6 months ago in view of bilateral tubovarian mass. Histopathological examination of the specimen obtained showed uterus with proliferative endometrium, chronic cervicitis and xanthogranulomatous salpingo oopheritis (left side). Right ovary was normal. On presentation to us, except for mild tenderness in the left side of lower abdomen, her physical examination was unremarkable. Computed tomography (CT) scan showed right sided hydroureteronephosis mixed density lesion measuring $5.8 \times 3.9 \mathrm{~cm}$ with few cystic enhancing solid components noted in right adnexa [Figure 1]. Right ureter could be traced upto this right adnexal lesion (suggestive of external compression from the adnexal lesion). Renal functions were normal. She underwent laparotomy with right oophorectomy. Right distal ureter was fibrotic so was excised and Boari flap was done. Histopathological diagnosis rendered was Xanthogranulomatous inflammation with abscess formation and chronic ureteritis with fibrosis [Figure 2]. Postoperative period was uneventful. Patient is asymptomatic in three months follow up.

\section{IDISCUSSION}

Xathogranulomatous inflammation of pelvis is an uncommon entity.
We have reported here two cases of the same, where the diagnosis was challenging. Though xathogranulomatous has a very clear cut picture on histopathological examination, the clinical picture can be misleading. Many cases in literature have been reported where it mimicked malignancy with invasion of surrounding tissue and markedly elevated tumor markers. ${ }^{[1,2]}$ However these cases which we encountered did not clinically confuse us with malignancy. Nese Yener et al. concluded in their report that frozen section is mandatory to rule out malignancy. ${ }^{[3]}$ We would like to add that xanthogranulomatous inflammation not always masquerades malignancy, so role of frozen section may be limited to selected cases only.

Though both cases we encountered had a pelvic mass of around $5 \mathrm{~cm}$. Shailaja Shukla et al. have reported huge cyst arising from pelvis and reaching the epigastrium $(15.6 \times 10.8$ $\mathrm{cm})$ which posed a dilemma of being a mucinous cystadenoma. ${ }^{[4]}$
Figure 1: Transabdominal ultrasound (a) and computed tomography (b) showing adnexal mass in case 2


Figure 2: Histopathology: 2a. Low power view showing xanthogranulomatous inflammation (2a1- case 1; 2a2- case 2); 2b Photomicrograph showing sheets of foamy macrophages interspersed with lymphocytes and few neutrophils (H\&E, 400X) (2b1- case 1; 2b2- case 2)
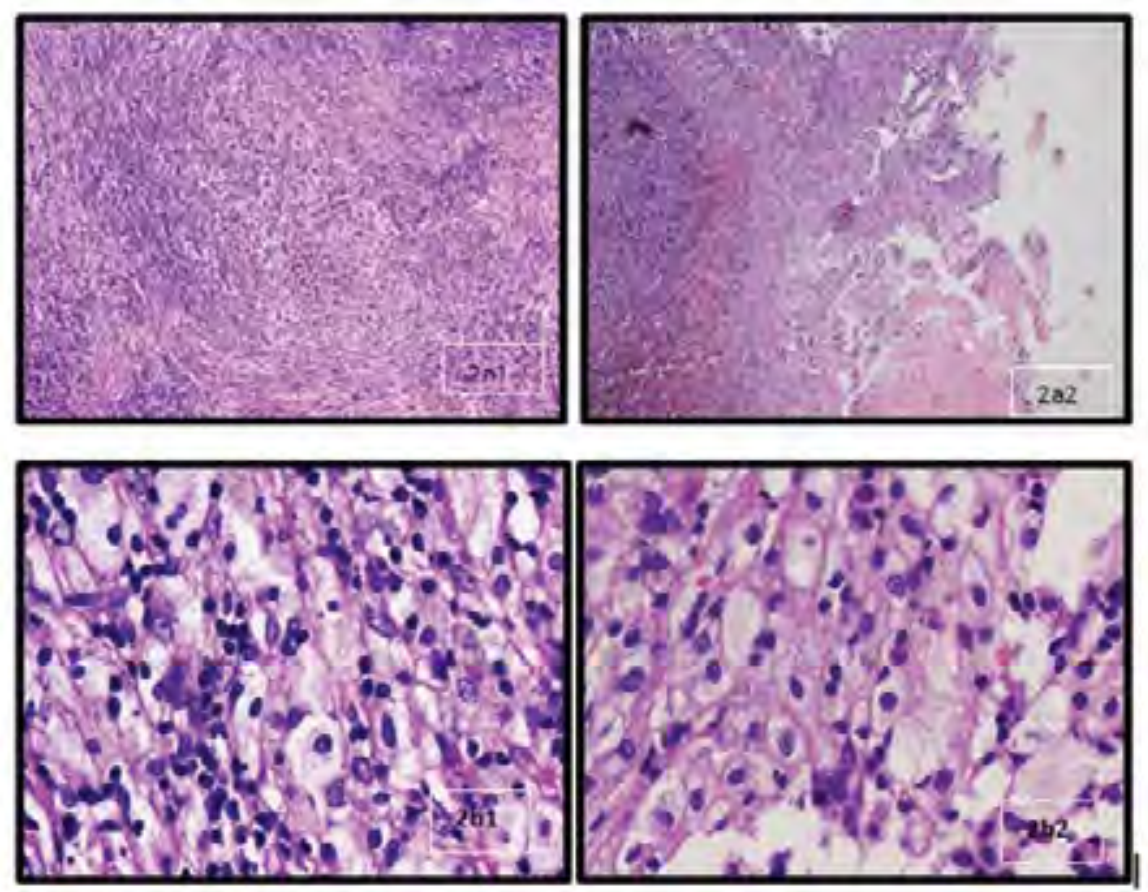
The second case reported here to our knowledge is the first in English literature of adnexal xanthogranulomatous causing ureteric obstruction.

First case of ours had a history of IUD usage for long time (8 years), similar to that reported by Yulia Gray at al. With the popularization of newer IUDs with long life span, should it be kept in mind as a rare complication. ${ }^{[5]}$

$\mathrm{n}$ few of the reports for confirmation of diagnosis immunohistiochemical stains were found to be useful. ${ }^{[2]}$ However in our experience which is supported by extensive available literature the histopathological picture of xanthogranulomatous under the microscope is quite clear most of the times. There is characteristic massive infiltration of the tissues by lipid-laden histiocytes admixed with inflammatory cells such as lymphocytes, plasma cells and polymorphonuclear leucocytes.
Xanthogranulomatous inflammtion is reported to be caused by ineptly treated PID. Among the various proposed etiopathologic mechanisms of xanthogranulomatous, is infection followed by ineffective antibiotic therapy. Inefficient or inappropriate antibiotics used in the early phase of infection which didn't control the multiplication of bacteria can lead to this. Macrophages phagocytize the lipid and change into foam cells. [2] We also suspect the same to be the probable mechanism in both of our cases. Hence, inadvertent use of antibiotics in suspected cases of PID where the woman is not responding as anticipated should be minimized.

\section{REFERENCES:}

1. Mila Ghosh, Puja Sakhuja and Anil $\mathrm{K}$ Agarwal. Xanthogranulomatous cholecystitis: a premalignant condition? Hepatobiliary Pancreat Dis Int. 2011;10:179-184.
2. Xiang-sheng Zhang, Hong-yan Dong, Leilei Zhang, Mohamed Mokhtar Desouki, Chengquan Zhao. Xanthogranulomatous Inflammation of the Female Genital Tract: Report of Three Cases. Journall of Cancer 2012; 3: 100-106.

3. Nese Yener, Erdin Ilter, Ahmet Midi. Xanthogranulomatous salpingitis as a rare pathologic aspect of chronic active pelvic inflammatory disease. Indian Journal of Pathology and Microbiology 2011; 54 : 141-143.

4. Shailaja Shukla, Mukta Pujani, Sanjeet Kumar Singh, Meenu Pujani. Xathogranulomatous oophoritis associated with primary infertility and endometriosis. Indian Journal of Pathology and Microbiology 2010; 53: 197-198

5. Yulia Gray, N. Peter Libbey. Xanthogranulomatous Salpingitis and Oophoritis: A Case Report and Review of the Literature. Arch Pathol Lab Med. 2001;125:260-263. 